\title{
ISSN: 2668-6384
}

https://conferenceproceedings.international

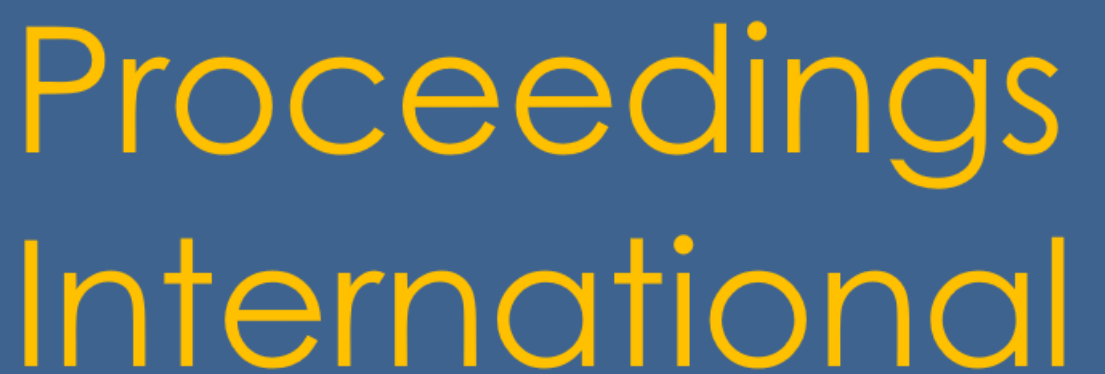

Volume 1, Issue 1, Pages 0036-0038 2019

Proceedings, Abstract

\section{Ultrastructural peculiarities in basal cell carcinoma}

\author{
A.-M. Moroşanu ${ }^{1}$, N. Mirancea ${ }^{1}$, F.D. Juravle ${ }^{2}$ \\ 1 Bucharest Institute of Biology, Romanian Academy, Bucharest, Romania \\ 2 Plastic and Aestethic Surgery Department, "MedLife" Orthopedy and Plastic Surgery Hospital, Bucharest, Romania \\ * Correspondence: anamaria.morosanu@ibiol.ro; Scopus ID: 55984580300
}

The last decade was marked by a statistically significant increase in the incidence of skin cancers, which motivated the development of new studies to later understand the behavior of these pathologies developing new therapeutic approaches. Also, the multitude of premalignant lesions as well as the complex classification of the carcinomas required a more accurate differentiation of the differential diagnosis, and in this regard the present electron microscopic study contributes significantly.

A tumor is a very complex ecosystem represented in particular by (1) genetically modified neoplastic cells and (2) tumor stroma represented by (a) various other cell types (fibroblasts, fibrocytes, mast cells, inflammatory cells, endothelial cells, myelinated or non-myelinated nerves, etc.), and (b) extracellular matrix (basal lamina, elastic fibers and collagen, but also soluble molecules) [1].

The purpose of this study was to discover new aspects of ultrastructure that occur in basal cell carcinoma cases investigated by us, related to the capacity of invasiveness of these tumors. Fresh tumor fragments were obtained with the informed consent of the patients. Here we present some peculiar aspects concerning infrastructure of tumor cells involved in invasive process, especially desmosomal and hemidesmosomal junctions, invadopodia and shedding membrane vesicles. Moreover, here we report about new described cell phenotype termed telocytes involved in cell signaling by their homo- and heterocellular contacts. Telocytes from basal cell carcinoma stroma exhibit a reduced number of heterocellular contacts, which suggests a possible perturbation of tissue homeostasis modulation.

Electron microscopic investigations revealed that in invasive basal cell carcinoma intercellular junctions, namely desmosomes are severely altered and that the tumor cells generate and disseminate membrane vesicles, including exosomes inside of the peritumoral stroma. Using transmission electron microscopy to investigate invasive basal cell carcinoma, we have managed to determine the relevance of all these changes for the purpose of evaluation of the invasive capacity of tumor cells within the peritumoral stroma.

Keywords: carcinoma, electron microscopy, basal cell carcinoma. 


\section{Ultrastructural peculiarities in basal cell carcinoma}

\section{Funding}

The study was supported by a project from the Institute of Biology Bucharest of Romanian Academy who took place during the period 2008-2011.

\section{Acknowledgments}

Not applicable.

\section{Conflicts of Interest}

The authors declare no conflict of interest.

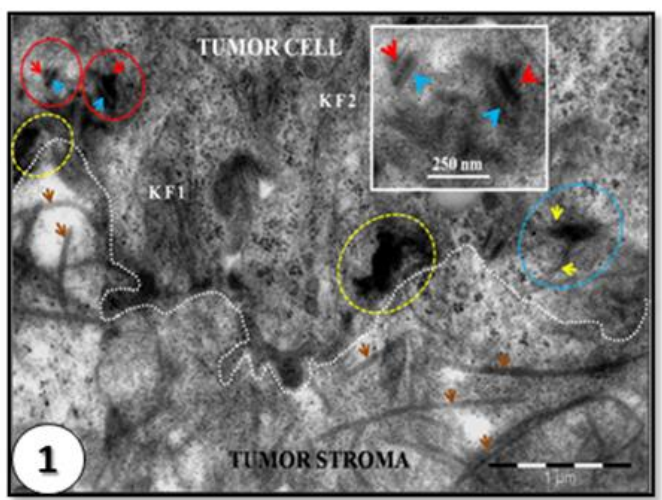

Figure 1 . The boundary between malignant keratinocytes and the peritumoral stroma is imprecise (white line dashed) as a result of the extensive dissolution of tumor cell plasma. At this level we see hemidesmosomal debris in the form of electronodense conglomerates (dotted yellow ellipses). Intracitoplasmic can be observed internalized desmosomes to which sometimes intermediate filaments of cytokeratin are attached (yellow arrow points in the dotted blue ellipse). Most often, internalized desmosomes debris can be identified as desmosomal plates (inset - red and blue arrows) at which does not attach intermediate cytokeratin filaments (dotted red circles).

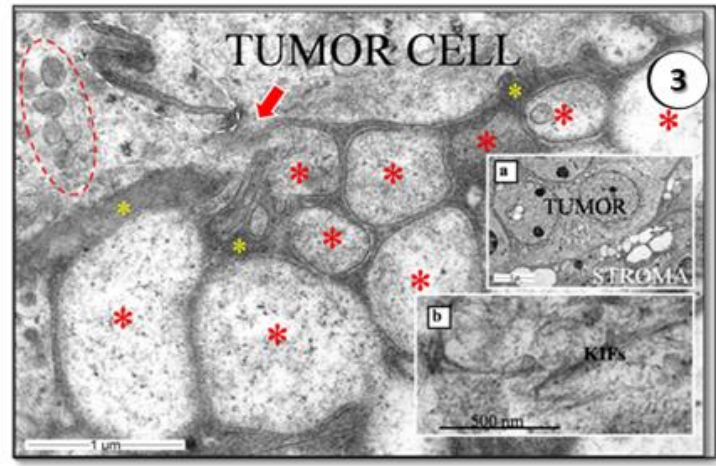

Figure 3. Between tumor cells at the stroma interface, desmosomal junctions (white elliptic area) are missing. A tumor cell has microvesicles (the red elliptic area). Shedding membrane vesicles released (red asterisks) can be observed. The basement membrane is missing, but dense amorphous material (yellow asterisks) is deposited. No hemidesmosomes can be detected. A space in the tumor cell plasma membrane (large arrow) is visible. Overview, inset a. Tumor cells still express intermediate keratin filaments (inset b, KIFs).

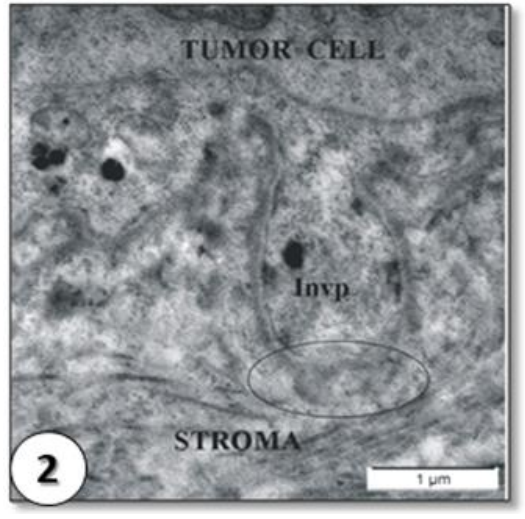

Figure 2. At the stroma-tumor interface, hemidesmosomes are almost missing. An invadopodium (Invp) is detached from a tumor cell. At this level, the plasma membrane as well as the basal lamina exhibit a discontinuity (ellipsoid area) [2].

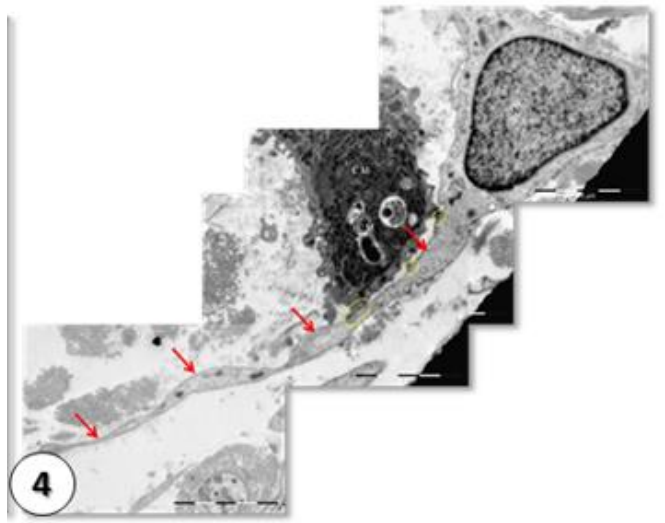

Figure 4. Heterotypic relationship (yellow circles) between a nucleated telocyte $(\mathrm{N})$, established between its telopode and a macrophage. 


\section{References}

1. Mirancea, N.; Mirancea, D.; Juravle, F.D.; . Şerban, A.M.; Mirancea, G.V. Epithelial-stromal interactions during tumorigenesis and invasion process of basocellular and squamous cell carcinomas at the tumorperitumoral stroma interfacerom. J. Biol. - Zool. 2009, 54, 97-120.
2. Mirancea, N.; Moroşanu, A.M.; Mirancea, G.V.; Juravle, F.D.; Mănoiu, V.S. Infrastructure of the telocytes from tumor stroma in the skin basal and squamous cell carcinomas. Rom. J. Morphol. Embryol. 2013, 54, 10251037.

(C) 2019 by the authors. This article is an open access article distributed under the terms and conditions of the Creative Commons Attribution (CC BY) license (http://creativecommons.org/licenses/by/4.0/). 